\title{
Depth-wise differentiation of Jones matrices obtained from Mueller optical coherence tomography
}

Milos Todorovic, Shuliang Jiao, George Stoica, Lihong V. Wang

Milos Todorovic, Shuliang Jiao, George Stoica, Lihong V. Wang, "Depthwise differentiation of Jones matrices obtained from Mueller optical coherence tomography," Proc. SPIE 5316, Coherence Domain Optical Methods and Optical Coherence Tomography in Biomedicine VIII, (1 July 2004); doi: $10.1117 / 12.531024$

SPIE. Event: Biomedical Optics 2004, 2004, San Jose, CA, United States 


\title{
Depth-wise differentiation of Jones matrices obtained from Mueller optical coherence tomography
}

\author{
Miloš Todorovića ${ }^{\mathrm{a}}$, Shuliang Jiao ${ }^{\mathrm{a}}$, George Stoica ${ }^{\mathrm{b}}$, Lihong V. Wang ${ }^{* a}$ \\ a Optical Imaging Laboratory, Department of Biomedical Engineering, Texas A\&M University, \\ College Station, TX 77843-3120 \\ ${ }^{\mathrm{b}}$ Department of Pathobiology, Texas A\&M University, College Station, TX 77843-5547
}

\begin{abstract}
A unique feature of polarization-sensitive Mueller optical coherence tomography (Mueller-OCT) is that it can reveal various polarization properties of biological samples that are not observable using conventional OCT. One of the most important polarization parameters is birefringence, which can be measured in its integrated form using existing Mueller-OCT systems. We present a new method that uses the least squares algorithm to differentiate measured integrated Jones matrices so that the samples can be observed layer-by-layer. We tested the algorithm using simulated data with variable additive white Gaussian noise (AWGN) levels. We further verified the algorithm using in vitro measurements of the porcine tendon and the septum of the rat heart. This least squares-based algorithm has the potential to reveal structures previously hidden by the inherent masking properties of the integrated images and provide localized phase retardation and orientation information.
\end{abstract}

Keywords: differentiation, polar decomposition, polarization, OCT, birefringence, orientation, phase retardation, Jones matrix, Mueller matrix.

\section{INTRODUCTION}

Ever since its introduction, optical coherence tomography (OCT) has been establishing itself as one of the most promising noninvasive imaging modalities offering high resolution and multiple contrast mechanisms. In addition to conventional OCT, which uses the amplitude based contrast, several other branches of OCT were developed based on detecting changes in polarization of light ${ }^{1-5}$ or Dopler shifts ${ }^{6-8}$ resulting from the flow of fluids. The contrast in an OCT image can result from the optical properties of a sample that modify the amplitude or the polarization state of the incident light field.

Birefringence is a description of the anisotropy of the phase velocity of light in a sample. It is inherent in a variety of biological components such as collagen, keratin, myelin, and elastic fibers. Many denaturalization processes, such as thermal denaturalization, alter birefringence in biological tissues and polarization-based OCT (PS-OCT) systems can detect these changes ${ }^{9-11}$. Hence, the ability to detect the birefringent properties of structures within tissues can enhance diagnosis.

Jiao et al have shown ${ }^{4}$ that a scattering sample acts as a nondepolarizing medium because of the heterodyne detection scheme used in OCT. Therefore, the polarization properties of biological samples can be described equally by either Jones or Mueller matrices. In order to provide complete information about polarization properties of a sample, PS-OCT should measure one of those matrices. We used the fiber-based polarization-sensitive Mueller-OCT system ${ }^{12}$ developed in the Optical Imaging Laboratory at Texas A\&M University to obtain Jones matrices used in this paper.

Previous papers ${ }^{2,4}$ reporting on birefringent properties of biological tissues used Jones and Mueller calculus to extract information about phase retardation and orientation from integrated round-trip matrices. Those matrices can give an insight into combined values of parameters of interest but cannot characterize individual layers precisely. We propose a new differentiation algorithm that is capable of extracting single-trip Jones matrices of individual layers. It is based on polar decomposition of matrices and employs a least squares algorithm to extract parameters, such as phase

*LWang@tamu.edu; http://oilab.tamu.edu 
retardation and orientation of the optical axes, from Jones matrices. We have tested the algorithm using both simulated data and in vitro measurements.

\section{DIFFERENTIATION ALGORITHM}

The measured Jones matrices along one A-scan obtained from Mueller-OCT encompass the integrated effect of all of the layers preceding the layer whose local polarization properties we are interested in. Therefore, the $i$-th measured Jones matrix from any A-scan is influenced by all preceding layers starting from the sample surface up to, and including, the $i$-th layer as shown in equation (1),

$$
\begin{aligned}
& \mathbf{J}_{e m 1}=\mathbf{J}_{s t 1}^{T} \mathbf{J}_{s t 1}=\mathbf{J}_{r t 1}, \\
& \mathbf{J}_{e m 2}=\mathbf{J}_{s t 1}^{T} \mathbf{J}_{s t 2}^{T} \mathbf{J}_{s t 2} \mathbf{J}_{s t 1}=\mathbf{J}_{s t 1}^{T} \mathbf{J}_{r t 2} \mathbf{J}_{s t 1}, \\
& \mathbf{J}_{e m 3}=\mathbf{J}_{s t 1}^{T} \mathbf{J}_{s t 2}^{T} \mathbf{J}_{s t 3}^{T} \mathbf{J}_{s t 3} \mathbf{J}_{s t 2} \mathbf{J}_{s t 1}=\mathbf{J}_{s t 1}^{T} \mathbf{J}_{s t 2}^{T} \mathbf{J}_{r t 3} \mathbf{J}_{s t 2} \mathbf{J}_{s t 1} .
\end{aligned}
$$

In equation (1), indices emi denote the expected measured matrices; rti denote the round-trip matrices of a single layer; and sti represent the one-way or actual Jones matrices of a given layer. We can recover every round-trip single-layer matrix by recovering one-way single-layer matrices one by one and eliminating their effects on measured matrices of deeper layers, as shown by a generalized formula

$$
\mathbf{J}_{r t i}=\left(\mathbf{J}_{s t(i-1)}^{T}\right)^{-1} \ldots\left(\mathbf{J}_{s t 1}^{T}\right)^{-1} \mathbf{J}_{e m i}\left(\mathbf{J}_{s t 1}\right)^{-1} \ldots\left(\mathbf{J}_{s t(i-1)}\right)^{-1}
$$

We need to assume a model of a tissue layer to proceed with characterizing its properties. A sequence of a linear retarder and linear diattenuator approximates a thin layer of biological tissue well. It is assumed that the fast axes of the retarder and diattenuator are collinear. The polar decomposition theorem, when used with Jones matrices, states that any non-depolarizing polarization element described by a Jones matrix can be represented as a cascade of a diattenuator and a retarder ${ }^{13}$. The polar decomposition yields two matrices - a unitary matrix $\mathbf{U}$ corresponding to a retarder and a nonnegative definite Hermitian matrix $\mathbf{H}$ corresponding to a diattenuator. Once round-trip matrices of individual layers are calculated using equation (2), we can apply the polar decomposition to obtain matrices $\mathbf{U}$ and $\mathbf{H}$. In order to calculate parameters of an individual layer we fitted in the least-squares sense the matrices $\mathbf{U}$ and $\mathbf{H}$ to the model Jones matrices of a linear retarder and diattenuator, respectively.

Equations (3) represent final expressions for calculating the orientation, retardation, and transmission parameters of Jones matrices of individual layers consisting of a sequence of a retarder and diattenuator.

$$
\begin{aligned}
& \tan 2 \theta=\frac{U_{m 12}^{i}+U_{m 21}^{i}}{U_{m 11}^{i}-U_{m 22}^{i}} \\
& \cot \varphi=\frac{U_{m 11}^{r}+U_{m 22}^{r}}{\left(U_{m 11}^{i}-U_{m 22}^{i}\right) \cos 2 \theta+\left(U_{m 12}^{i}+U_{m 21}^{i}\right) \sin 2 \theta} \\
& p_{x}=\sqrt{p_{x}{ }^{\prime}}=\sqrt{H_{m 11}^{r} \cos ^{2} \theta+H_{m 22}^{r} \sin ^{2} \theta+\left(H_{m 12}^{r}+H_{m 21}^{r}\right) \sin \theta \cos \theta} \\
& p_{y}=\sqrt{p_{y}{ }^{\prime}}=\sqrt{H_{m 11}^{r} \sin ^{2} \theta+H_{m 22}^{r} \cos ^{2} \theta-\left(H_{m 12}^{r}+H_{m 21}^{r}\right) \sin \theta \cos \theta},
\end{aligned}
$$


where $\varphi$ is the phase retardation; $\theta$ is the orientation angle of the fast eigenvector for both the diattenuator and retarder; and $p_{x}$ and $p_{y}$ are the transmittances of the fast and slow eigenvectors, respectively.

\section{RESULTS}

To evaluate the validity of the algorithm, we conducted a computer simulation with an artificially generated data set. A white Gaussian noise of different power levels was added to the signal in order to mimic the actual experimental environment more accurately. Figure 1 depicts the ability of the algorithm to recover the original values for retardation when the generated data set had SNR levels of 20 and $50 \mathrm{~dB}$. It is obvious that the number of layers that can be recovered successfully drops as the SNR worsens. We can see in Figure 1.a that the algorithm achieved a perfect recovery of the phase retardation values for noise-free and $50 \mathrm{~dB}$ SNR cases; when the SNR dropped to $20 \mathrm{~dB}$, the algorithm was able to recover approximately first 35 layers with little or no error. Even more illustrative is Figure 1.b where we show differences between the simulated retardation values and values obtained using the differentiation algorithm. Little or no deviation from the original values is observed for noise-free and $50 \mathrm{~dB}$ SNR. The only obvious departure occurs for SNR of $20 \mathrm{~dB}$ after the $35^{\text {th }}$ layer. SNR levels above $20 \mathrm{~dB}$ can be achieved in present day OCT systems, especially in the most superficial layers of biological samples. Therefore, this differentiation algorithm can perform correctly in actual systems.

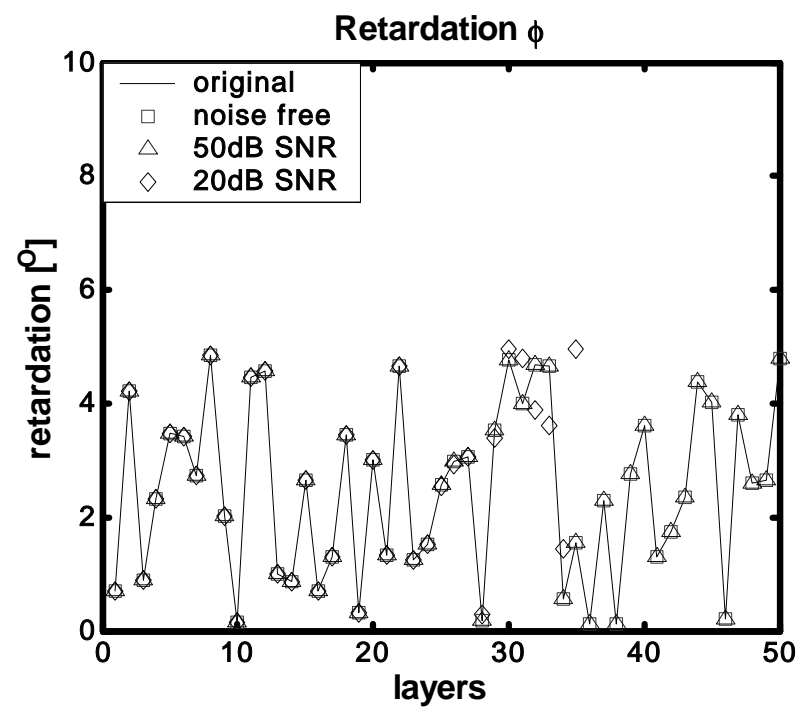

a.

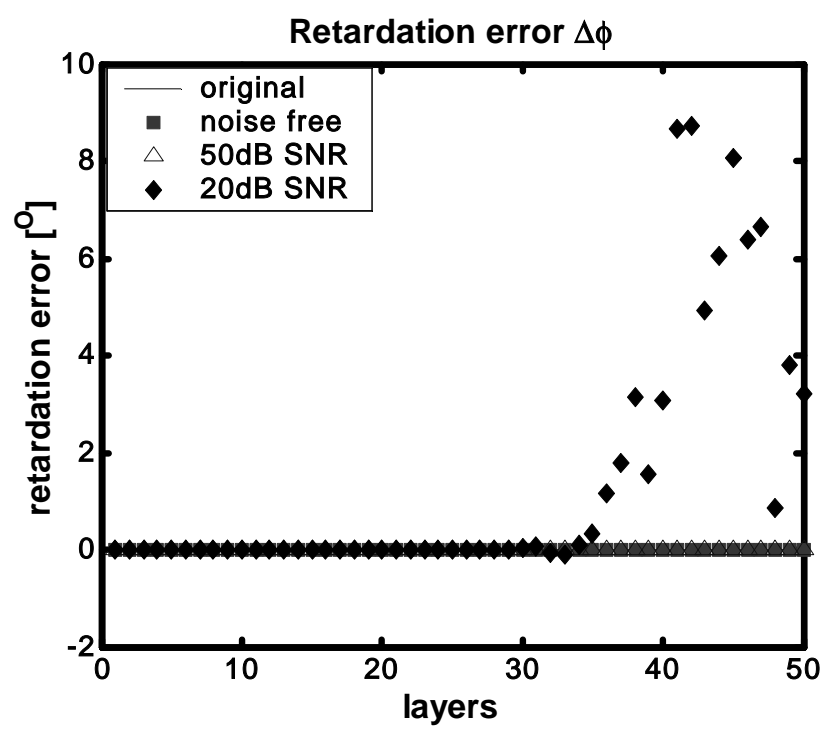

b.

Figure 1. Simulation results: a. recovered phase retardation - original phase retardation and retardation recovered for noise free and $50 \mathrm{~dB}$ SNR environments overlap; b. deviations from the original values.

In order to show the achievability of extracting parameters of one-way Jones matrices the algorithm was applied to calculate polarization parameters of biological samples. Tendon is the one of the samples that is extensively used to show the ability of OCT to image birefringence in biological tissue. Its highly pronounced birefringence originates from the high concentration of collagen fibers. The integrated phase retardation image of a typical tendon shows a regularly banded structure, which is a result of the 180 degrees phase wrapping. We imaged the fresh porcine tendon using the Mueller-OCT system that will be reported in the paper by our group ${ }^{12}$. The algorithm for calculating Jones matrices ${ }^{12}$ and the dynamic calibration technique for eliminating the effects of birefringence of the single mode fiber ${ }^{14}$ can be found in references. Figure 2 shows the integrated and differentiated phase retardation images together with the profiles of phase retardation along one A-scan. Integrated image was obtained from the measured Jones matrices without eliminating the effects of preceding layers. We can observe the banded structure in the integrated image and the phase 
retardation values span the range between 0 and 180 degrees. This clearly indicates that the previously mentioned 180 degrees phase wrapping is present in the image. On the other hand, differentiated image obtained using the algorithm presented in this paper is much flatter; there are regions with more pronounced retardation but the range of retardation values is only between 0 and 50 degrees, with majority of pixels having values up to 10 degrees. Higher values are observed deeper in the sample where the signal-to-noise ratio (SNR) is much lower than in the superficial layers of the sample. Profile of the integrated phase retardation values in Figure 2.c shows the oscillatory nature resulting from phase wrapping while the profile of the differentiated values does not exhibit any abrupt changes.
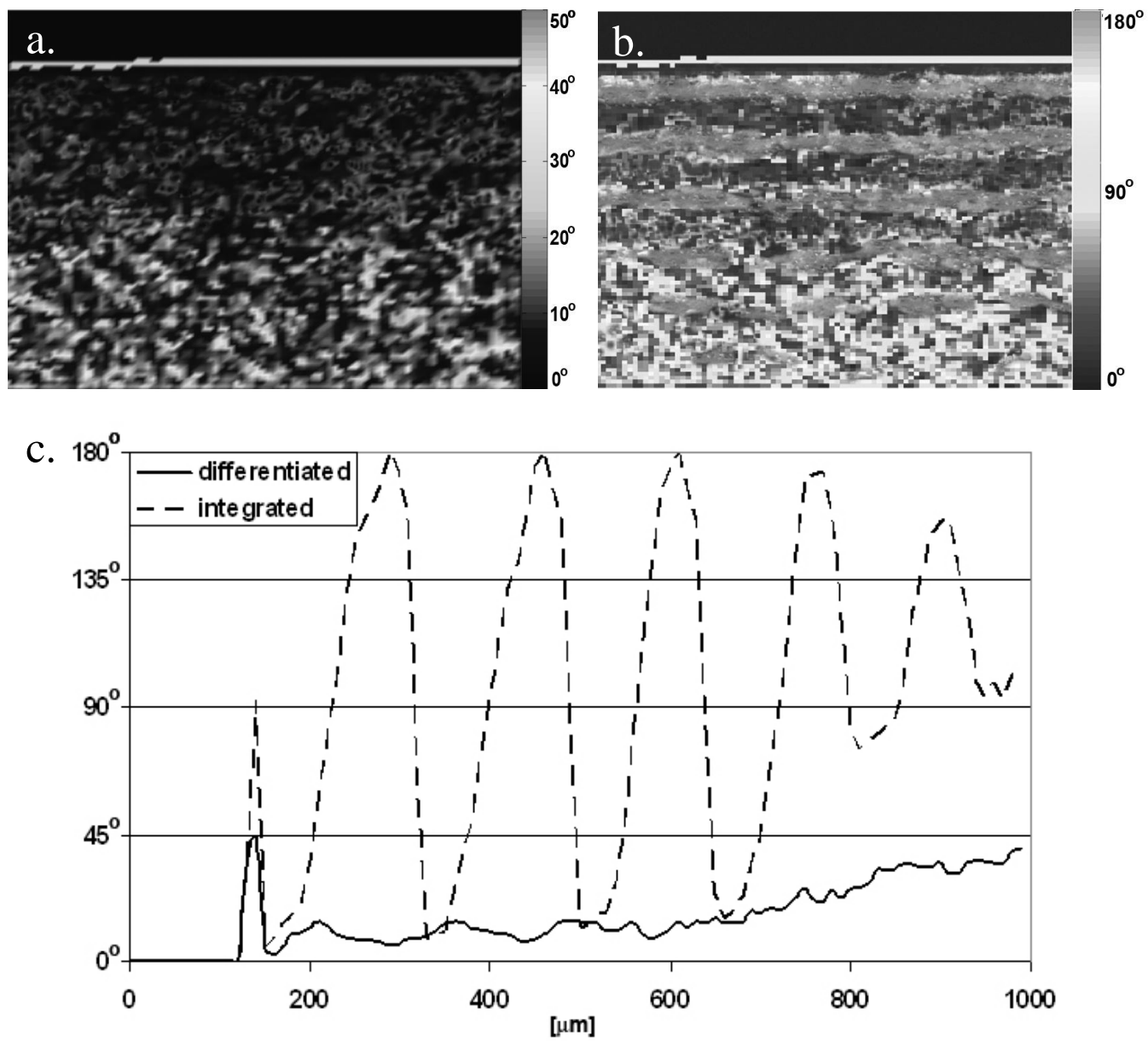

Figure 2. Phase retardation of the porcine tendon: a. differentiated image; b. integrated image; c. profiles of integrated and differentiated retardation along one averaged A-scan. Banded structure in the integrated phase retardation image results from 180 degrees phase wrapping. There is no phase wrapping present in the differentiated image. Image dimensions are $1 \mathrm{~mm} \times 1 \mathrm{~mm}$ (width $\mathrm{x}$ height).

Finally, we investigated the orientation of muscle fibers in the septum of the rat heart. The heart tissue was fixed in $10 \%$ buffered neutral formalin before imaging. Following imaging, the paraffin-embedded tissue was sectioned at 20 microns and stained with hematoxylin and eosin (HE). The stained sections were examined under an Olympus light microscope with $40 \mathrm{X}$ magnification. Figure 3 presents results of the analysis of the septum from the surface up to the depth of $680 \mu \mathrm{m}$ along one A-scan. In order to improve SNR, the A-scan used to calculate the orientation and phase retardation is a result of averaging over $50 \mathrm{~A}$-scans taken at the same spot on the surface of the septum. Orientation of fibers was observed from the histological images at different depths and is plotted in Figure 3 using a solid line. We can see that the calculated values are in good agreement (within standard deviation) with the data from histology. As expected, standard deviation increases with depth and this can be attributed to lower SNR in the deeper regions of the sample. The linear change of orientation observed in our study agrees with the results of a study ${ }^{15}$ where the authors observed that fiber orientation linearly changes from the surface of the septum towards the deeper layers. 


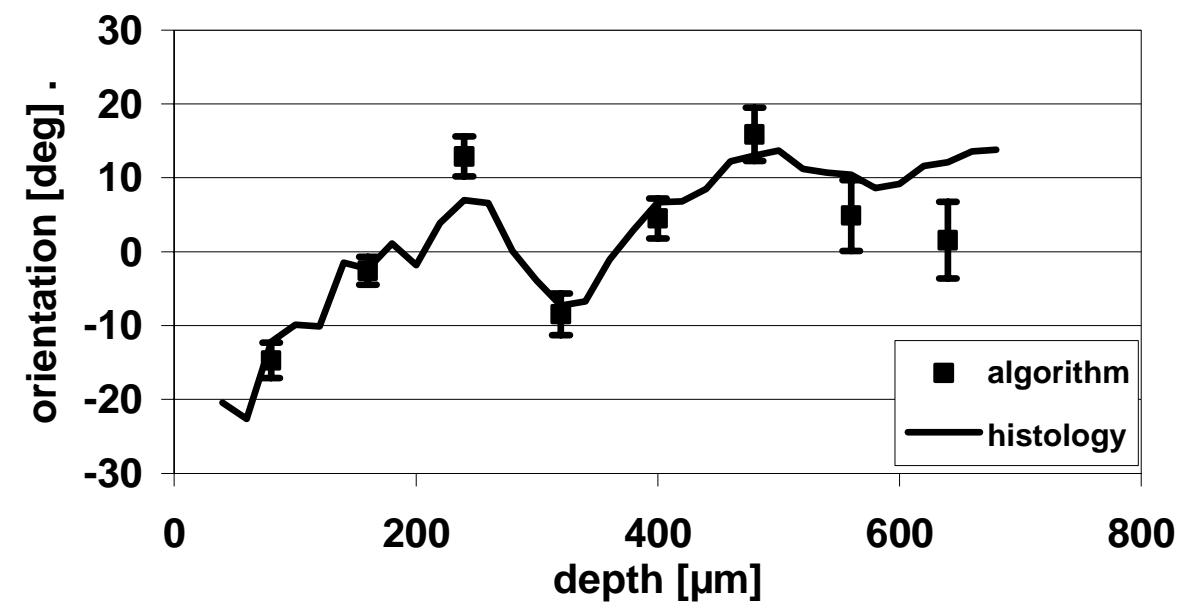

Figure 3. Orientation of muscle fibers in the septum of the rat heart. Data points from histological images are spaced at $20 \mu \mathrm{m}$ intervals while the data points obtained using the differentiation algorithm are $80 \mu \mathrm{m}$ apart.

\section{CONCLUSIONS}

Polarization properties of samples that do not depolarize incident light can be characterized either by Jones or Mueller matrices. Because of the detection scheme used in OCT, scattering samples such as biological tissue can be considered a nondepolarizing medium. Jones matrices fully characterize polarization properties, such as birefringence and diattenuation, of a sample. Changes in values of magnitude and orientation of these polarization parameters provide polarization-based contrast in PS-OCT. Previously reported algorithms for calculating the phase retardation and orientation of birefringence worked on integrated round-trip Jones matrices that are a direct outcome of OCT reconstruction algorithms.

In this work, we introduced a novel algorithm that uses polar decomposition and least squares fitting to recover single-trip Jones matrices of individual layers within scattering media. The algorithm starts with the round-trip Jones matrix of the most superficial layer and eliminates the effect of preceding layers on the consecutive round-trip matrices. This enables the use of polar decomposition, which decomposes the round-trip matrix of each layer into a unitary matrix corresponding to a retarder and a nonnegative definite Hermitian matrix corresponding to a diattenuator. Each layer is assumed to be a series of a linear retarder and diattenuator. Although the accuracy of the algorithm heavily depends on the SNR level, in its current form it is capable of extracting useful information from the depths up to a half millimeter in the sample. Future work will include the implementation of a de-noising algorithm that should aid the differentiation by increasing the SNR.

We have shown the capabilities of this method using the computer simulation and in vitro measurements of the porcine tendon and the septum of the rat heart. OCT images of the differentiated phase retardation have shown that we were able to eliminate the integrating effect that is present in images where phase retardation is calculated from integrated round-trip Jones matrices. We were also able to recover the orientation of muscle fibers in the septum. With further improvements, this depth-wise differentiation algorithm promises to enhance the polarization-based contrast in OCT by revealing structures previously hidden by the inherent masking properties of the integrated images.

\section{ACKNOWLEDGMENTS}

This project was sponsored by the National Institutes of Health grant R01 CA092415.

\section{REFERENCES}

1. M.R. Hee, D. Huang, E.A. Swanson and J.G. Fujimoto, "Polarization-sensitive low-coherence reflectometer for birefringence characterization and ranging," Journal of Optical Society of America B, 9, 903-908, 1992. 
2. J.F. de Boer, T.E. Milner, M.J.C. van Gemert and J.S. Nelson, "Two-dimensional birefringence imaging in biological tissue by polarization-sensitive optical coherence tomography," Optics Letters, 22, 934-936, 1997.

3. S. Jiao, G. Yao and L.V. Wang, "Depth-resolved two-dimensional Stokes vectors of backscattered light and Mueller matrices of biological tissue measured with optical coherence tomography," Applied Optics, 39, 6318$6324,2000$.

4. S. Jiao and L.V. Wang, "Two-dimensional depth-resolved Mueller matrix of biological tissue measured with double-beam polarization-sensitive optical coherence tomography," Optics Letters, 27, 101-103, 2002.

5. S. Jiao and L.V. Wang, "Jones-matrix imaging of biological tissues with quadruple-channel optical coherence tomography," Journal of Biomedical Optics, 7, 350-358, 2002.

6. J.A. Izatt, M.D. Kulkarni, S. Yazdanfar, J.K. Barton and A.J. Welsh, "In vivo bidirectional color Doppler flow imaging of picoliter blood volumes using optical coherence tomography," Optics Letters, 22, 1439-1441, 1997.

7. M.C. Pierce, B.H. Park, B. Cense and J.F. de Boer, "Simultaneous intensity, birefringence, and flow measurements with high-speed fiber-based optical coherence tomography," Optics Letters, 27, 1534-1536, 2002.

8. H. Ren, Z. Ding, Y. Zhao, J. Miao, J.S. Nelson and Z. Chen, "Phase-resolved functional optical coherence tomography: simultaneous imaging of in situ tissue structure, blood flow velocity, standard deviation, birefringence, and Stokes vectors in human skin," Optics Letters, 27, 1702-1704, 2002.

9. K. Schoenenberger, B.W. Colston, D.J. Maitland, L.B. Da Silva and M.J. Everett, "Mapping of birefringence and thermal damage in tissue by use of polarization-sensitive optical coherence tomography," Applied Optics, 37, 60266036, 1998.

10. J.F. de Boer, S.M. Srinivas, A. Malekafzali, Z. Chen and J.S. Nelson, "Imaging thermally damaged tissue by polarization sensitive optical coherence tomography," Optics Express, 3, 212-218, 1998.

11. S. Jiao, W. Yu, G. Stoica and L.V. Wang, "Phase-based polarization contrast in Mueller optical coherence tomography and application in burn imaging," Applied Optics, 42, 5191-5197, 2003.

12. S. Jiao, M. Todorović, G. Stoica and L.V. Wang, "Fiber-based Mueller-matrix optical coherence tomography with continuous source polarization modulation", in preparation

13. J.J. Gil and E. Bernabeu, "Obtainment of the polarizing and retardation parameters of a non-depolarizing optical system from the polar decomposition of its Mueller matrix," Optik, 76, 67-71, 1987.

14. S. Jiao, W. Yu, G. Stoica and L.V. Wang, "Optical-fiber-based Mueller optical coherence tomography," Optics Letters, 28, 2003.

15. P.M.F. Nielsen, I.J. Le Grice, B.H. Smaill and P.J. Hunter, "Mathematical model of geometry and fibrous structure of the heart," American Journal of Physiology, 260, H1365-H1378, 1991. 